\title{
Chapter 10 \\ Optimal Control Strategies for an Advertisement Viral Diffusion
}

\author{
João N. C. Gonçalves, Helena Sofia Rodrigues \\ and M. Teresa T. Monteiro
}

\begin{abstract}
The process of diffusing viral marketing campaigns through social networks can be modeled under concepts of mathematical epidemiology. Based on a Susceptible-Infected-Recovered (SIR) epidemiological model, the benefits of optimal control theory on the diffusion of a real viral advertisement are studied. Two optimal control strategies that could help marketers to maximize the spread of information and minimize the costs associated to it in optimal time windows are analyzed and compared. The uniqueness of optimality system is proved. Numerical simulations show that high investment costs in publicity strategies do not imply high overall levels of information diffusion. This paper contributes to the current literature by studying a viral marketing campaign using real numerical data.
\end{abstract}

Keywords Optimal control theory $\cdot$ Viral marketing $\cdot$ SIR epidemiological model $\cdot$ Information diffusion strategies

\subsection{Introduction}

Marketing is a valuable tool to orient and increase the performance of a company [15]. However, traditional marketing strategies are having difficulty to meet demanding conditions of consumers [13]. Therefore, to create competitive advantages against other companies, marketing professionals have been trying to design attractive and

J. N. C. Gonçalves $(\bowtie) \cdot$ M. T. T. Monteiro

Department of Production and Systems, Algoritmi R\&D Center,

University of Minho, Braga, Portugal

e-mail: jncostagoncalves@gmail.com

M. T. T. Monteiro

e-mail: tm@dps.uminho.pt

H. S. Rodrigues

School of Business Studies, Polytechnic Institute of Viana do Castelo, Valença, Portugal

e-mail: sofiarodrigues@esce.ipvc.pt

H. S. Rodrigues

Department of Mathematics, Center for Research and Development in Mathematics

and Applications (CIDMA), University of Aveiro, Aveiro, Portugal 
viral campaigns, based on strategies that allow to revert the drawbacks of traditional marketing. One of these strategies is known as Viral Marketing (VM), which refers to the process that takes advantage of word-of-mouth to replicate and diffuse a marketing message into a large set of customers [9]. VM aims to reach a large audience with a low cost associated to it, by exploiting network effects which in turn maximize information diffusion. Moreover, VM has proved to be a sustainable marketing strategy by avoiding the necessity of establishing a direct contact with targeted individuals [11]. Nonetheless, some downsides can be highlighted to VM, such as its uncontrollable nature or even the difficulty in controlling the timing and success of the transmission phenomena (see [19] and the references cited therein).

Optimal control theory is an extension of the calculus of variations that seeks to find control strategies for a dynamic system [12], and gives some insights on its usefulness by optimizing marketing strategies that maximize the spread of information without losing much money.

Over time, optimal control problems applied to marketing have been proposed and discussed (see, e.g., [4-6] and the references cited therein). More precisely, these research studies focus on study optimal strategies to promote information diffusion in social networks and environments.

Considering that VM can be modeled by epidemiological models [9, 14, 17], this article studies the dynamics and impact of a real VM advertisement - Dove Real Beauty Sketches, based on real data presented in [18]. Produced in 2013, Dove Real Beauty Sketches is a publicity campaign that focuses on state the definition of beautiful, promoting self-esteem and changing the perception of beauty [2]. However, one of the major difficulties in diffusing a viral message relates to create mechanisms that convince and persuade individuals to spread it in the best time window. Thus, by choosing this campaign as an example of how to build a successful marketing strategy, our aim is to study optimal policies and time intervention strategies that could help marketing professionals to increase information diffusion with low cost in future campaigns. For that, a controlled SIR epidemiological model proposed in [4] is analyzed and applied to Dove's advertisement. Additionally, some numerical simulations related to different investment cost scenarios are performed.

The paper is organized as follows. Section 10.2 presents the mathematical problem according to the marketing campaign real data. In Sect. 10.3, an optimal control problem is formulated and studied. Section 10.4 presents numerical simulations related to the comparison of different marketing strategies and guidelines regarding the best control policies to apply in different investment scenarios. Conclusions are carried out in Sect. 10.5.

\subsection{SIR Epidemiological Model and Properties}

In this section, the SIR epidemic model without control is formulated and basic properties within a marketing context are established. This model subdivides the population into three mutually-exclusive compartments: susceptible individuals, 
who correspond to the portion of the target population who can spread the marketing message $(S)$; infected individuals, who correspond to the set of population who encourage the spreading of the message among social networks $(I)$; recovered individuals, who stop diffusing the marketing message $(R)$.

The dynamics, over time $t$, of the mutually-exclusive compartments can be described by the following system of ordinary differential equations:

$$
\left\{\begin{array} { l l } 
{ \frac { d S ( t ) } { d t } = - \beta \frac { S ( t ) I ( t ) } { N } } \\
{ \frac { d I ( t ) } { d t } = \beta \frac { S ( t ) I ( t ) } { N } - \gamma I ( t ) } \\
{ \frac { d R ( t ) } { d t } = \gamma I ( t ) , }
\end{array} \left\{\begin{array}{l}
S(0)=S_{0}>0 \\
I(0)=I_{0}>0 \\
R(0)=0 .
\end{array}\right.\right.
$$

The total population and its rate of change at time $t$ are given by

$$
N=S+I+R \Leftrightarrow \frac{d S}{d t}+\frac{d I}{d t}+\frac{d R}{d t}=0, \forall t \in[0, \infty) .
$$

Hence, since $R(t)=N-S(t)-I(t)$, the system (10.1) can be reduced to

$$
\left\{\begin{array}{l}
\frac{d S(t)}{d t}=-\beta \frac{S(t) I(t)}{N} \\
\frac{d I(t)}{d t}=\beta \frac{S(t) I(t)}{N}-\gamma I(t) .
\end{array}\right.
$$

Exploring the dynamics of the system (10.3), when the marketing message is appealing, individuals leave the class $S$ and move to class $I$ at a rate $\beta$. Over time, individuals in the class $I$ stop sharing the message and move to class $R$ at a rate $\gamma$. It should be emphasized that the variables $S(t), I(t)$ and $R(t)$, parameters $\beta$ and $\gamma$, and initial conditions are non-negative. Thus, it can be shown that the solutions of the SIR system are also non-negative (see, e.g., [3]).

Henceforth, further analyses consider a selected set of parameter values, in part computed based on numerical algorithms described in the literature and in part taken from real numerical data related to Dove's campaign (see Table 10.1). It is also appropriate to reformulate the model (10.3) in terms of the fractions of $S, I$ and $R$, by considering $s=\frac{S}{N}, i=\frac{I}{N}, r=\frac{R}{N}$.

Using FMINSEARCH routine (see [7]) from MATLAB optimization toolbox, included in the algorithm proposed in [10], parameters estimation was performed based on the first seven days of Dove's marketing campaign, where the maximum peak of infection is attained. Obtained by the inequality $\frac{d i(t)}{d t}>0$ for $s \approx 1$, the basic reproduction number of the normalized SIR model $\left(\mathscr{R}_{0}=\frac{\beta}{\gamma}\right)$ expresses the number of secondary transmissions made by a single infected individual, within a susceptible set of popu- 
Table 10.1 Parameters and initial conditions

\begin{tabular}{l|l|l|l}
\hline Symbol & Description & Estimated value & References \\
\hline$\beta$ & $\begin{array}{l}\text { Predisposition to share the } \\
\text { marketing message }\end{array}$ & 67.5244 & {$[10]$} \\
\hline$\gamma$ & $\begin{array}{l}\text { Cessation of diffusion of the } \\
\text { marketing message }\end{array}$ & 65.0751 & {$[10]$} \\
\hline$N$ & $\begin{array}{l}\text { Total population at the campaign } \\
\text { launching }\end{array}$ & $10^{9}$ & {$[16]$} \\
\hline$S(0)$ & $\begin{array}{l}\text { Number of susceptible individuals } \\
\text { at the campaign launching }\end{array}$ & $10^{9}-30.000$ & {$[18]$} \\
\hline$I(0)$ & $\begin{array}{l}\text { Number of infected individuals at } \\
\text { the campaign launching }\end{array}$ & 30.000 & {$[18]$} \\
\hline$R(0)$ & $\begin{array}{l}\text { Number of recovered individuals at } \\
\text { the campaign launching }\end{array}$ & 0 & {$[18]$} \\
\hline
\end{tabular}

lation [14]. It can be proved that if $\mathscr{R}_{0}<1$ the marketing message is not widespread. However, in this case, $\mathscr{R}_{0}>1$, which confirms that Dove's campaign was a viral epidemic (see, e.g., [3] for more details on the dynamics of $\mathscr{R}_{0}$ in this model).

\subsection{Optimal Control Problem}

As proposed in [4], an optimal control problem related to the model analyzed so far is formulated. After the normalization of the model (10.3), two control functions $u_{1}(t)$ and $u_{2}(t), \forall t \in[0,6]$, are added. Hence, considering $r(t)=1-s(t)-i(t)$, the resultant state system of ordinary differential equations with optimal control is given by

$$
\begin{cases}\frac{d s(t)}{d t}=-\left(\beta+u_{2}(t)\right) s(t) i(t)-u_{1}(t) s(t) & \left\{\begin{array}{l}
s(0)=\frac{S(0)}{N} \\
\frac{d i(t)}{d t}=\left(\beta+u_{2}(t)\right) s(t) i(t)+u_{1}(t) s(t)-\gamma i(t),
\end{array}\right. \\
i(0)=\frac{I(0)}{N} .\end{cases}
$$

The control functions $u_{1}(t)$ and $u_{2}(t)$ express the recruitment of susceptible individuals to act as spreaders within the targeted population (e.g., via advertisements in mass media) and the encouragement of infected individuals to continue to spread the marketing message into their social network (e.g., through vouchers, rewards, monetary stimuli), respectively [4].

Let $t_{f}$ be the considered campaign deadline. The set of admissible control functions is defined as

$$
\Omega=\left\{\left(u_{1}(\cdot), u_{2}(\cdot)\right) \in\left(L^{2}\left(0, t_{f}\right)\right)^{2} \mid 0 \leqslant u_{1}(t) \leqslant u_{1 \max } \wedge 0 \leqslant u_{2}(t) \leqslant u_{2 \max }, \forall t \in\left[0, t_{f}\right]\right\} .
$$


For $u_{i}: i \in\{1,2\}$, note that the application of the control policy is maximum if $u_{i}=u_{i \max }$, and minimum if $u_{i}=0$. The main objective is to find the optimal values $u_{1}^{*}$ and $u_{2}^{*}$ for the controls $u_{1}$ and $u_{2}$, in such a way that the state trajectories $s$ and $i$ are the solution of the system (10.4) over $\left[0, t_{f}\right]$ and maximize the objective functional (10.5). As a trade-off, the optimal control problem consists in maximize the spreading of information at the final time and minimize the intervention costs related to the application of the control policies, i.e.,

$$
\max _{\Omega} J\left(u_{1}(\cdot), u_{2}(\cdot)\right)=r\left(t_{f}\right)+i\left(t_{f}\right)+\int_{0}^{t_{f}}-\left[B u_{1}^{2}(t)+C u_{2}^{2}(t)\right] d t
$$

subject to (10.4), where the non-negative constants $B$ and $C$ represent the weights of the investment cost associated to the control signals $u_{1}$ and $u_{2}$, respectively. The quadratic structure of the weighted controls is consistent with related literature (see, e.g., $[4,8])$. The existence of the optimal controls can be proved using existence results of optimal solutions studied in [1].

Under the Pontryagin's Maximum Principle (PMP) [12] and considering the optimal control problem (10.4) and objective functional $(10.5)$, if $\left(u_{1}^{*}(\cdot), u_{2}^{*}(\cdot)\right)$ is a control pair that is optimal for the problem, then there exists a nontrivial Lipschitz continuous mapping, called adjoint vector, $\lambda:\left[0, t_{f}\right] \rightarrow \mathbb{R}^{2}, \lambda(t)=\left(\lambda_{1}(t), \lambda_{2}(t)\right)$, such that

$$
\frac{d s}{d t}=\frac{\partial H}{\partial \lambda_{1}}, \quad \frac{d i}{d t}=\frac{\partial H}{\partial \lambda_{2}}
$$

and

$$
\frac{d \lambda_{1}}{d t}=-\frac{\partial H}{\partial s}, \quad \frac{d \lambda_{2}}{d t}=-\frac{\partial H}{\partial i}
$$

where the function $H$ defined by

$$
\begin{aligned}
H\left(s(t), i(t), u_{1}(t), u_{2}(t), \lambda_{1}(t), \lambda_{2}(t)\right) & =-\left(B u_{1}^{2}(t)+C u_{2}^{2}(t)\right) \\
& +\lambda_{1}(t)\left[-\left(\beta+u_{2}(t)\right) s(t) i(t)-u_{1}(t) s(t)\right] \\
& +\lambda_{2}(t)\left[\left(\beta+u_{2}(t)\right) s(t) i(t)+u_{1}(t) s(t)-\gamma i(t)\right]
\end{aligned}
$$

is called the Hamiltonian. At time $t$, let $s^{*}$ and $i^{*}$ be the optimal state trajectories. Thus, according to the PMP it follows that

$$
\left\{\begin{array}{l}
\frac{d \lambda_{1}}{d t}=\lambda_{1}(t)\left[\left(\beta+u_{2}^{*}(t)\right) i^{*}(t)+u_{1}^{*}(t)\right]-\lambda_{2}(t)\left[\left(\beta+u_{2}^{*}(t)\right) i^{*}(t)+u_{1}^{*}(t)\right] \\
\frac{d \lambda_{2}}{d t}=\lambda_{1}(t)\left[\left(\beta+u_{2}^{*}(t)\right) s^{*}(t)\right]-\lambda_{2}(t)\left[\left(\beta+u_{2}^{*}(t)\right) s^{*}(t)-\gamma\right],
\end{array}\right.
$$


with transversality conditions $\lambda_{1}\left(t_{f}\right)=0$ and $\lambda_{2}\left(t_{f}\right)=1$, since $r\left(t_{f}\right)$ and $i\left(t_{f}\right)$ appear as payoffs terms in the objective functional (10.5).

In addition, by setting $\frac{\partial H}{\partial u_{1}}$ and $\frac{\partial H}{\partial u_{2}}$ to zero and due to the boundedness of the control functions $u_{1}(t)$ and $u_{2}(t)$ on $\Omega$, the optimal controls $u_{1}^{*}(t)$ and $u_{2}^{*}(t)$ are characterized by:

$$
u_{1}^{*}(t)=\min \left\{\max \left\{\frac{s^{*}(t)\left(\lambda_{2}(t)-\lambda_{1}(t)\right)}{2 B}, 0\right\}, u_{1 \max }\right\},
$$

and

$$
u_{2}^{*}(t)=\min \left\{\max \left\{\frac{s^{*}(t) i^{*}(t)\left(\lambda_{2}(t)-\lambda_{1}(t)\right)}{2 C}, 0\right\}, u_{2 \max }\right\} .
$$

At this point, it is possible to derive the optimality system, consisting of the state system (10.4), the adjoint system (10.6) and transversality conditions with the characterizations (10.7) and (10.8).

Theorem 10.1 (Uniqueness of Optimality System) Given the initial value problem (10.4) and the objective functional (10.5), the optimal solution $\left(s^{*}(t), i^{*}(t)\right)$ with associated optimal control functions $u_{1}^{*}(t), u_{2}^{*}(t)$ and the adjoint functions $\lambda_{1}(t)$, $\lambda_{2}(t)$ are unique for $t_{f}$ sufficiently small.

Proof Let $\left(s, i, \lambda_{1}, \lambda_{2}\right)$ and $\left(\bar{s}, \bar{i}, \overline{\lambda_{1}}, \overline{\lambda_{2}}\right)$ be two solutions of the optimality system.

Consider $s=e^{\phi t} a_{1}, i=e^{\phi t} a_{2}, \lambda_{1}=e^{-\phi t} b_{1}, \lambda_{2}=e^{-\phi t} b_{2}$. Analogously, consider $\bar{s}=e^{\phi t} \overline{a_{1}}, \bar{i}=e^{\phi t} \overline{a_{2}}, \overline{\lambda_{1}}=e^{-\phi t} \overline{b_{1}}, \overline{\lambda_{2}}=e^{-\phi t} \overline{b_{2}}$, where $\phi$ is a constant.

Let

$$
\begin{gathered}
u_{1}(t)=\min \left\{\max \left\{\frac{a_{1}\left(b_{2}-b_{1}\right)}{2 B}, 0\right\}, u_{1 \max }\right\}, \\
u_{2}(t)=\min \left\{\max \left\{\frac{e^{\phi t} a_{1} a_{2}\left(b_{2}-b_{1}\right)}{2 C}, 0\right\}, u_{2 \max }\right\},
\end{gathered}
$$

and

$$
\begin{gathered}
\overline{u_{1}}(t)=\min \left\{\max \left\{\frac{\overline{a_{1}}\left(\overline{b_{2}}-\overline{b_{1}}\right)}{2 B}, 0\right\}, u_{1 \max }\right\}, \\
\overline{u_{2}}(t)=\min \left\{\max \left\{\frac{e^{\phi t} \overline{a_{1} a_{2}}\left(\overline{b_{2}}-\overline{b_{1}}\right)}{2 C}, 0\right\}, u_{2 \max }\right\} .
\end{gathered}
$$


Henceforth, for the sake of simplicity, it will be considered $u_{1}, \overline{u_{1}}, u_{2}, \overline{u_{2}}$ instead of the above characterizations. Using these assumptions, the first state equation of the optimality system becomes

$$
e^{\phi t} \frac{d a_{1}}{d t}+\phi e^{\phi t} a_{1}=-\left(\beta+u_{2}\right) e^{2 \phi t} a_{1} a_{2}-u_{1} e^{\phi t} a_{1} .
$$

Now, the equations for $s$ and $\bar{s}, i$ and $\bar{i}, \lambda_{1}$ and $\overline{\lambda_{1}}, \lambda_{2}$ and $\overline{\lambda_{2}}$ are subtracted. Then, each of these equations is multiplied by an appropriate difference of functions and integrated from 0 to $t_{f}$. Next, the four integral equations are added and some estimates are performed. Below, we illustrate one of these manipulations using the Eq. (10.9).

$$
\begin{aligned}
& \frac{1}{2}\left(a_{1}\left(t_{f}\right)-\overline{a_{1}}\left(t_{f}\right)\right)^{2}+\phi \int_{0}^{t_{f}}\left(a_{1}-\overline{a_{1}}\right)^{2} d t \\
= & \left.-\int_{0}^{t_{f}} e^{\phi t}\left[\left(\beta+u_{2}\right) a_{1} a_{2}-\left(\beta+\overline{u_{2}}\right) \overline{a_{1} a_{2}}\right)\right]\left(a_{1}-\overline{a_{1}}\right) d t-\int_{0}^{t_{f}}\left(u_{1} a_{1}-\overline{u_{1} a_{1}}\right)\left(a_{1}-\overline{a_{1}}\right) d t \\
= & -\int_{0}^{t_{f}} e^{\phi t}\left[\beta\left(\left(a_{1}-\overline{a_{1}}\right) a_{2}+\overline{a_{1}}\left(a_{2}-\overline{a_{2}}\right)\right)+\left(u_{2}-\overline{u_{2}}\right) a_{1} a_{2}+\overline{u_{2}}\left(a_{1}-\overline{a_{1}}\right) a_{2}\right. \\
& \left.+\overline{u_{2} a_{1}}\left(a_{2}-\overline{a_{2}}\right)\right]\left(a_{1}-\overline{a_{1}}\right) d t-\int_{0}^{t_{f}}\left[\left(u_{1}-\overline{u_{1}}\right) a_{1}+\overline{u_{1}}\left(a_{1}-\overline{a_{1}}\right)\right]\left(a_{1}-\overline{a_{1}}\right) d t \\
\leqslant & D \int_{0}^{t_{f}}\left(a_{1}-\overline{a_{1}}\right)^{2}+\left(b_{1}-\overline{b_{1}}\right)^{2}+\left(b_{2}-\overline{b_{2}}\right)^{2} d t+E e^{\phi t_{f}} \int_{0}^{t_{f}}\left(a_{1}-\overline{a_{1}}\right)^{2}+\left(a_{2}-\overline{a_{2}}\right)^{2} d t \\
& +F e^{3 \phi t_{f}} \int_{0}^{t_{f}}\left(b_{1}-\overline{b_{1}}\right)^{2}+\left(b_{2}-\overline{b_{2}}\right)^{2} d t,
\end{aligned}
$$

where $D, E$ and $F$ are constants. After estimate all the four equations of the optimality system, and noting that $e^{\phi t_{f}} \leqslant e^{3 \phi t_{f}}$, all integral equations are combined producing the following inequality:

$$
\begin{aligned}
& \frac{1}{2}\left[\left(a_{1}\left(t_{f}\right)-\overline{a_{1}}\left(t_{f}\right)\right)^{2}+\left(a_{2}\left(t_{f}\right)-\overline{a_{2}}\left(t_{f}\right)\right)^{2}+\left(b_{1}(0)-\overline{b_{1}}(0)\right)^{2}+\left(b_{2}(0)-\overline{b_{2}}(0)\right)^{2}\right] \\
& \quad+\phi \int_{0}^{t_{f}}\left(a_{1}-\overline{a_{1}}\right)^{2}+\left(a_{2}-\overline{a_{2}}\right)^{2}+\left(b_{1}-\overline{b_{1}}\right)^{2}+\left(b_{2}-\overline{b_{2}}\right)^{2} d t \\
& \leqslant \widetilde{D} \int_{0}^{t_{f}}\left(a_{1}-\overline{a_{1}}\right)^{2}+\left(a_{2}-\overline{a_{2}}\right)^{2}+\left(b_{1}-\overline{b_{1}}\right)^{2}+\left(b_{2}-\overline{b_{2}}\right)^{2} d t \\
& \quad+\widetilde{F} e^{3 \phi t_{f}} \int_{0}^{t_{f}}\left(a_{1}-\overline{a_{1}}\right)^{2}+\left(a_{2}-\overline{a_{2}}\right)^{2}+\left(b_{1}-\overline{b_{1}}\right)^{2}+\left(b_{2}-\overline{b_{2}}\right)^{2} d t .
\end{aligned}
$$

Rearranging the terms, the result is

$$
\begin{aligned}
& \frac{1}{2}\left[\left(a_{1}\left(t_{f}\right)-\overline{a_{1}}\left(t_{f}\right)\right)^{2}+\left(a_{2}\left(t_{f}\right)-\overline{a_{2}}\left(t_{f}\right)\right)^{2}+\left(b_{1}(0)-\overline{b_{1}}(0)\right)^{2}+\left(b_{2}(0)-\overline{b_{2}}(0)\right)^{2}\right] \\
& \leqslant\left(\widetilde{D}+\widetilde{F} e^{3 \phi t_{f}}-\phi\right) \int_{0}^{t_{f}}\left(a_{1}-\overline{a_{1}}\right)^{2}+\left(a_{2}-\overline{a_{2}}\right)^{2}+\left(b_{1}-\overline{b_{1}}\right)^{2}+\left(b_{2}-\overline{b_{2}}\right)^{2} d t,
\end{aligned}
$$


where $\widetilde{D}$ and $\widetilde{F}$ depend on the coefficients and the bounds of $a_{1}, a_{2}, b_{1}, b_{2}$. By choosing $\phi>\widetilde{D}+\widetilde{F}$ and $t_{f}<\frac{1}{3 \phi} \log \left(\frac{\phi-\widetilde{D}}{\widetilde{F}}\right)$, it therefore follows that

$$
\begin{aligned}
0 & \leqslant \frac{1}{2}\left[\left(a_{1}\left(t_{f}\right)-\overline{a_{1}}\left(t_{f}\right)\right)^{2}+\left(a_{2}\left(t_{f}\right)-\overline{a_{2}}\left(t_{f}\right)\right)^{2}+\left(b_{1}(0)-\overline{b_{1}}(0)\right)^{2}+\left(b_{2}(0)-\overline{b_{2}}(0)\right)^{2}\right] \\
& \leqslant\left(\widetilde{D}+\widetilde{F} e^{3 \phi t_{f}}-\phi\right) \int_{0}^{t_{f}}\left(a_{1}-\overline{a_{1}}\right)^{2}+\left(a_{2}-\overline{a_{2}}\right)^{2}+\left(b_{1}-\overline{b_{1}}\right)^{2}+\left(b_{2}-\overline{b_{2}}\right)^{2} d t \\
& \leqslant 0
\end{aligned}
$$

which implies that

$$
\begin{aligned}
& \left(\widetilde{D}+\widetilde{F} e^{3 \phi t_{f}}-\phi\right) \int_{0}^{t_{f}}\left(a_{1}-\overline{a_{1}}\right)^{2}+\left(a_{2}-\overline{a_{2}}\right)^{2}+\left(b_{1}-\overline{b_{1}}\right)^{2}+\left(b_{2}-\overline{b_{2}}\right)^{2} d t=0 . \\
& \text { Thus, knowing that }\left(\widetilde{D}+\widetilde{F} e^{3 \phi t_{f}}-\phi\right)<0 \text {, we have } a_{1}=\overline{a_{1}}, a_{2}=\overline{a_{2}}, b_{1}=\overline{b_{1}}, \\
& b_{2}=\overline{b_{2}} \text {, and }\left(s, i, \lambda_{1}, \lambda_{2}\right)=\left(\bar{s}, \bar{i}, \overline{\lambda_{1}}, \overline{\lambda_{2}}\right) .
\end{aligned}
$$

Remark 10.1 Since the state system (10.4) is autonomous, the proof of theorem 10.1 holds for any time $t_{f}$.

\subsection{Numerical Results and Discussion}

In this section, the influence of the optimal control strategies incorporated in the SIR model (10.4) with objective functional (10.5) is studied. The main goal is to provide insights related to when and which control strategies should be applied to maximize the spreading of information and minimize costs.

Using MATLAB software to solve the optimality system, numerical results were obtained using a forward-backward fourth-order Runge-Kutta scheme. More detailed information on this numerical scheme is presented in [8]. Numerical simulations consider a campaign deadline $t_{f}=6$, which corresponds to the Dove's campaign data on the first seven days.

In what follows, two approaches are considered. Firstly, in Sect. 10.4.1, simulations of the control weights are performed using both optimal controls $u_{1}^{*}$ and $u_{2}^{*}$, in order to assess which control weights induce a higher objective functional. Secondly, in Sect. 10.4.2, these pairs are used to model and compare scenarios related to high and low investment costs in publicity actions. 


\subsubsection{Simulation of the Control Weights Using $u_{1}^{*}$ and $u_{2}^{*}$}

Firstly, using both optimal controls $u_{1}^{*}$ and $u_{2}^{*}$, the variation of the control weights on both infected individuals $(i(t))$ and control signals is studied over $[0,6]$. The simulations of the control weights are performed under three strategies described in Table 10.2.

The choice of the values for $B$ and $C$ was based on several experimental simulations that aimed at obtaining the results that best described the reality of each strategy. For the sake of consistency, since at the beginning of a viral epidemic the main goal is to attract susceptible individuals, it is conceivable to start with an intermediate investment cost in detriment of a lower one. For this reason, the above strategies do not consider $B<1$. The simulations regarding the three strategies are illustrated in Fig. 10.1. Figure 10.1a shows that around the first day, the fraction of infected individuals is higher when the investment costs in fostering people who had already been in contact with the marketing message are low, namely for $B=1$ and $C=10^{-3}$.

Regarding the Strategy 2 (Fig. 10.1b), by fixing $B=1$, as the control weight $C$ increases, infection levels do not vary. Thus, it is plausible to conclude that higher costs in implement further publicity strategies to foster infected individuals do not result in higher levels of spreading.

In what concerns the Strategy 3, Fig. 10.1c reports that as the control weight $B$ increases, the number of people who have contact with the intended message is diminishing all the time and the maximum peak of infection is attained increasingly late.

Transversally, whatever the strategy considered, the smaller the investment costs neither in fostering infected individuals to continue to spread the message, nor in recruit susceptible individuals to act as spreaders, the greater the levels of information spreading.

Overall, Table 10.3 presents the values for the objective functional (10.5) by varying the control weights for each strategy.

Table 10.2 Optimal control marketing strategies

\begin{tabular}{l|l}
\hline \# Strategy & Marketing context \\
\hline $\begin{array}{l}\text { Strategy } 1: B=1 \text { and } C \in\left\{x \mid x=10^{-i}\right. \\
\text { and } i=1, \ldots, 3\}\end{array}$ & $\begin{array}{l}\text { Low investment costs in encouraging infected } \\
\text { individuals to continue to spread the marketing } \\
\text { message (e.g., exploiting social networks such as } \\
\text { Facebook and Twitter) }\end{array}$ \\
\hline $\begin{array}{l}\text { Strategy 2:B=1 and } C \in\left\{x \mid x=10^{i}\right. \\
\text { and } i=1, \ldots, 3\}\end{array}$ & $\begin{array}{l}\text { High investment costs in encouraging infected } \\
\text { individuals to continue to spread the marketing } \\
\text { message (e.g., monetary rewards and stimuli, } \\
\text { expensive promotional gifts and international trips) }\end{array}$ \\
\hline $\begin{array}{l}\text { Strategy } 3: B \in\left\{x \mid x=10^{i}\right. \\
\text { and } i=0, \ldots, 3\} \text { and } C=1\end{array}$ & $\begin{array}{l}\text { Increasing the investment costs in recruiting } \\
\text { susceptible individuals to act as spreaders }\end{array}$ \\
\hline
\end{tabular}




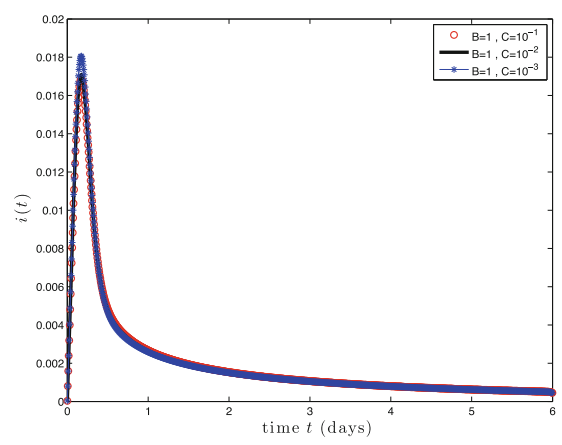

(a) $i(t)$ for Strategy 1

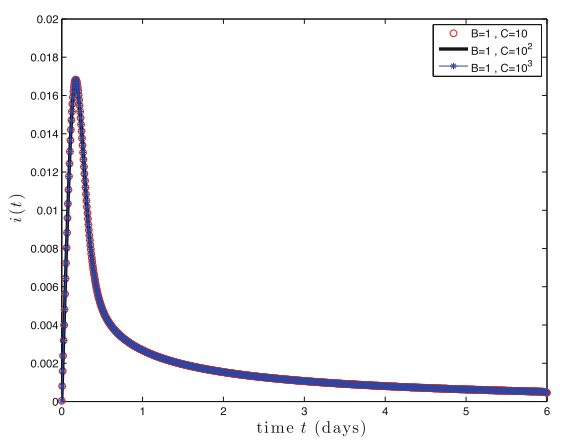

(b) $i(t)$ for Strategy 2

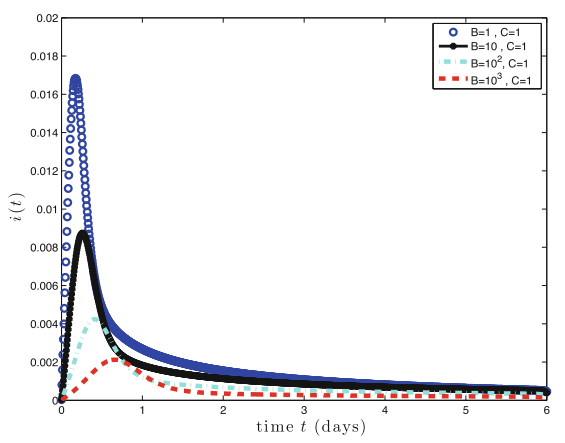

(c) $i(t)$ for Strategy 3

Fig. 10.1 Variation of the control weights on $i(t)$ for the different marketing strategies

Table 10.3 Summary of objective functionals varying control weights $(B, C)$

\begin{tabular}{l|l|l|l|l|l}
\hline \multicolumn{5}{l|}{ Strategy 1} & \multicolumn{2}{|c|}{ Strategy 2} & \multicolumn{2}{l}{ Strategy 3} \\
\hline$(B, C)$ & $\approx J\left(u_{1}^{*}, u_{2}^{*}\right)$ & $(B, C)$ & $\approx J\left(u_{1}^{*}, u_{2}^{*}\right)$ & $(B, C)$ & $\approx J\left(u_{1}^{*}, u_{2}^{*}\right)$ \\
\hline$\left(\mathbf{1}, \mathbf{1 0}^{-\mathbf{3}}\right)$ & $\mathbf{0 . 6 9 9 7 6 6}$ & $(\mathbf{1}, \mathbf{1 0})$ & $\mathbf{0 . 6 9 8 6 9 2}$ & $(\mathbf{1}, \mathbf{1})$ & $\mathbf{0 . 6 9 8 6 9 3}$ \\
\hline$\left(1,10^{-2}\right)$ & 0.698815 & $\left(1,10^{2}\right)$ & 0.698691 & $(10,1)$ & 0.463743 \\
\hline$\left(1,10^{-1}\right)$ & 0.698704 & $\left(1,10^{3}\right)$ & 0.698691 & $\left(10^{2}, 1\right)$ & 0.273901 \\
\hline- & - & - & - & $\left(10^{3}, 1\right)$ & 0.159022 \\
\hline
\end{tabular}

In Table 10.3, recalling the aim of maximize the objective functional (10.5), the pairs of control weights that induce a higher cost functional are highlighted in bold, for each strategy. The choice of the highlighted pairs was based on the aim of portray low, high and equal investment cost scenarios, respectively, to study their effects on the diffusion of the marketing message. These three scenarios are simulated in the next section. 


\subsubsection{Control Experiments for Different Investment Costs Scenarios}

In this Section, scenarios based on the control weights highlighted in Table 10.3 are simulated, varying the control functions $u_{1}(t)$ and $u_{2}(t), \forall t \in[0,6]$. If the company has monetary funds to invest in extra publicity actions either on susceptible or already infected individuals, upper control policies are tested. The upper control is defined as the maximum control value for which the cost functional (10.5) is positive, and it represents the maximum application of a control measure. Henceforth, let $u_{1 \text { max }}$ and $u_{2 \max }$ be the upper controls related to the control functions $u_{1}$ and $u_{2}$, respectively.

\subsubsection{Low Investment Costs Scenario Using Control Weights $(B, C)=\left(1,10^{-3}\right)$}

In Fig. 10.2a it is possible to note that the fraction of infected individuals is significantly higher whenever control is applied. In this regard, the implementation effect of the controls $u_{1}$ and $u_{2}$ is assessed, separately and combined, over [0, 6], see Fig. 10.3.

Observing Fig. 10.3a, b at the launch of the advertisement, the best policy is to implement the optimal control combination $\left(u_{1}^{*}, u_{2}^{*}\right)$, in order to rapidly attain the maximum peak of infection at the end of the first day of Dove's campaign (see Fig. 10.2a). Then, during the next 2 days, the control $u_{2}$ is at the upper bound (see, Fig. 10.3b), suggesting in this time window that the best policy is to apply $\left(0, u_{2}^{*}\right)$ in such a way as to encourage infected individuals to continue to spread the message. Hence, at the end of $t=2$, when the levels of recovery begin to increase, the pair $\left(u_{1}^{*}, 0\right)$ should be implemented in order to minimize the rate of recovered individuals by attracting susceptible individuals to diffuse the intended message, see Fig. 10.3a.

Notice that, in terms of the objective functional, despite of infection levels attain a maximum level using $\left(u_{1 \max }, u_{2 \max }\right), J\left(u_{1 \max }, u_{2 \max }\right) \approx 0.002095$, which means that a double investment in both control policies compromises the objective of mini-

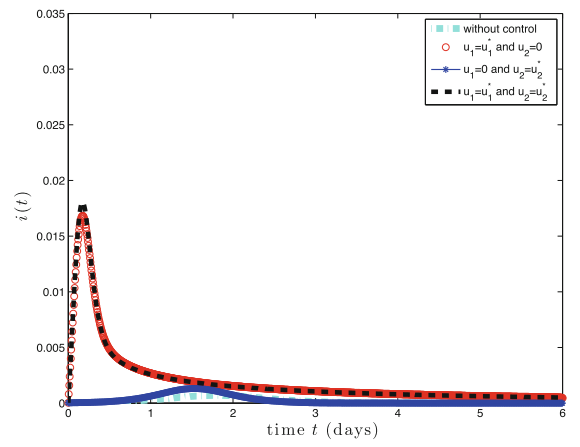

(a) Optimal controls

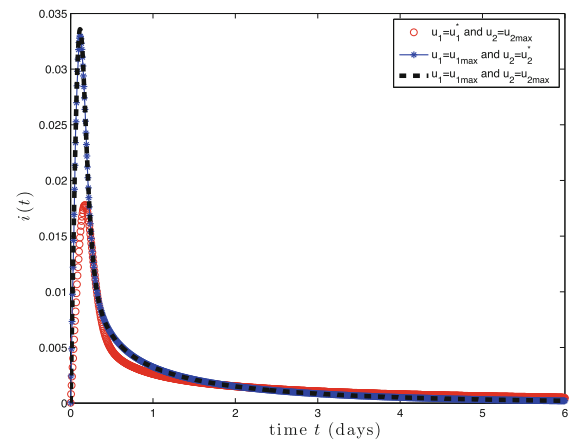

(b) Mixing upper and optimal controls

Fig. $10.2 i(t)$ with $B=1, C=10^{-3}, u_{1 \max }=0.4, u_{2 \max }=1$ 


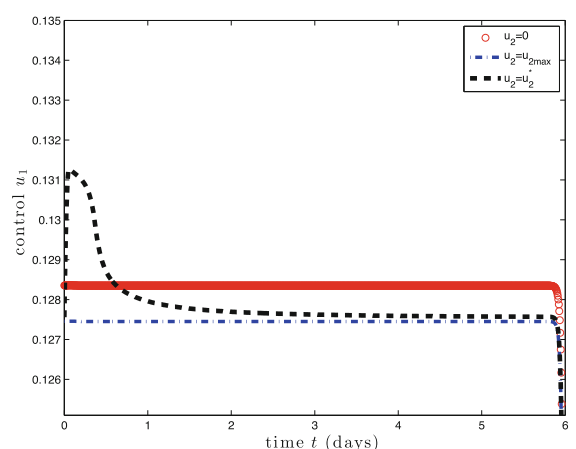

(a) Optimal control $u_{1}$

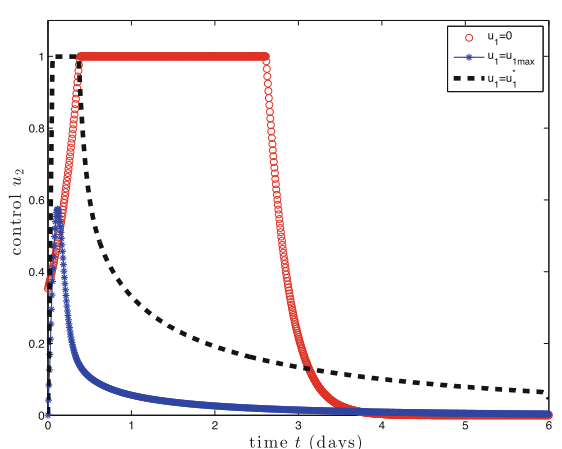

(b) Optimal control $u_{2}$

Fig. 10.3 Optimal controls $u_{1}$ and $u_{2}$ with $B=1, C=10^{-3}, u_{1 \max }=0.4, u_{2} \max =1$

mize costs. In contrast, based on Table $10.3, J\left(u_{1}^{*}, u_{2}^{*}\right) \approx 0.699766$, that is, the simultaneous use of the control functions $u_{1}^{*}$ and $u_{2}^{*}$ fulfills the proposed trade-off. This is underpinned by the fact that, for the control intervention $\left(u_{1}^{*}, u_{2 \max }\right)$, the cost functional (10.5) is almost the same as the obtained when the optimal controls $u_{1}^{*}$ and $u_{2}^{*}$ are applied $\left(J\left(u_{1}^{*}, u_{2 \max }\right) \approx 0.695835\right)$. These arguments show the importance of the control $u_{1}$ to attain the maximum peak of infection at the beginning of the campaign.

\subsubsection{High Investment Costs Scenario Using Control Weights $(B, C)=(1,10)$}

In this scenario, analogously to the previous one, the fraction of infected individuals is higher with the implementation of control policies than without it, see Fig. 10.4a.

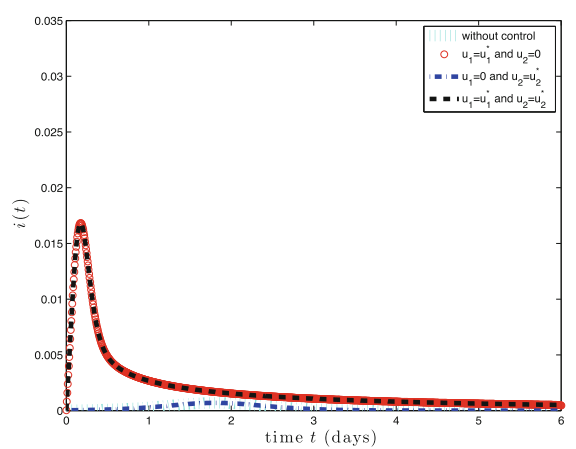

(a) Optimal controls

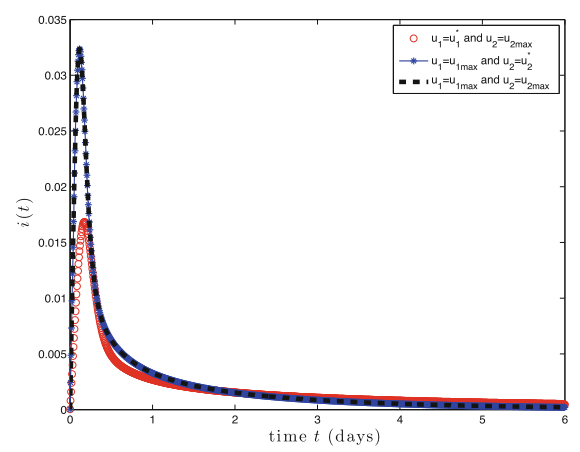

(b) Mixing upper and optimal controls

Fig. 10.4 $i(t)$ with $B=1, C=10, u_{1 \max }=0.4, u_{2} \max =0.01$ 


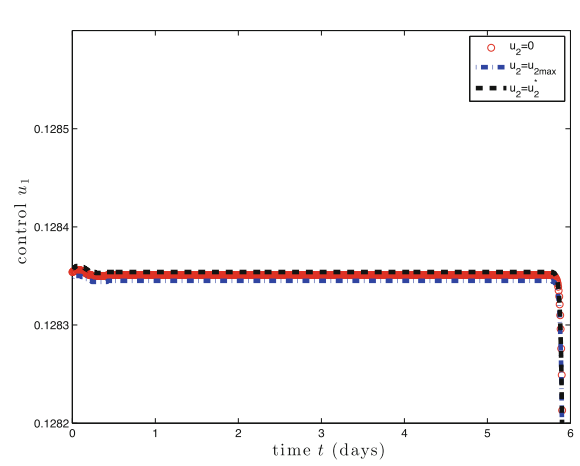

(a) Optimal control $u_{1}$

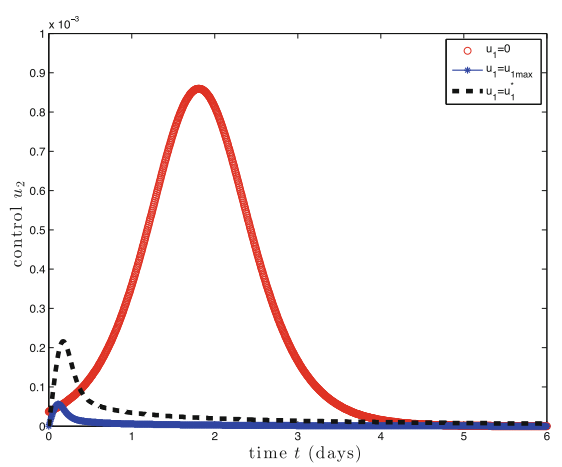

(b) Optimal control $u_{2}$

Fig. 10.5 Optimal controls $u_{1}$ and $u_{2}$ with $B=1, C=10, u_{1 \max }=0.4, u_{2} \max =0.01$

However, Fig. 10.5 illustrates that the magnitude of the control $u_{2}$ is significantly lower than the magnitude of $u_{1}$. By linking this finding with Fig. 10.4a, it is possible to infer not only that the optimal control measure $u_{2}^{*}$ applied by itself has no direct influence on the information dissemination, but also that by applying $\left(u_{1}^{*}, 0\right)$, the levels of information diffusion are satisfactory.

When upper control measures are applied, the infection levels increase substantially, see Fig. 10.4b. However, the use of $\left(u_{1 \max }, u_{2 \max }\right)$ results in a residual objective functional $\left(J\left(u_{1 \max }, u_{2 \max }\right) \approx 0.001591\right)$. At this point, upper control policies for $u_{2}$ are disadvantageous, inasmuch as the adoption $\left(u_{1}^{*}, u_{2 \max }\right)$ leads to the same infection levels as the obtained by using both optimal controls and $J\left(u_{1}^{*}, u_{2}^{*}\right) \approx 0.698692$, see Fig. 10.4 a, b. Furthermore, $J\left(u_{1}^{*}, 0\right) \approx 0.698678$, which means that the control $u_{1}^{*}$ is a sufficient condition to achieve the proposed trade-off.

Analogously to the previous strategy, it also can be noticed that the control $u_{1}$ has much more influence on the information spreading than $u_{2}$.

In order to avoid redundancy, the control simulations using $B=C=1$ were omitted. However, in this case, $u_{1}$ has much more influence on the information spreading than $u_{2}$. In addition, to meet the objective functional, optimal control strategies overlap the efforts imposed by upper control policies.

\subsection{Conclusion}

This paper applies optimal control theory to a real viral marketing campaign, by using real numerical data. The uniqueness of optimality system is proved. We show that the spreading of information attains high levels of dissemination at much faster rates when optimal control theory is applied. It is observed that when $B$ is fixed and $C$ decreases, the levels of information diffusion tend to grow up. In contrast, when $B$ is fixed and $C$ increases, the levels of information spreading do not vary and are lower than the values obtained for smaller levels of $C$. 
In order to improve the timing of the information diffusion, we recommend, for each scenario, optimal time windows to apply the control policies $u_{1}$ and $u_{2}$. Moreover, we conclude that the infection transmission process is maximized by using optimal control policies related to $u_{1}$ and $u_{2}$. However, it should be noted that due to the chaotic and quasi-unpredictable nature of viral campaigns, the success of a campaign depends on a constant monitoring and controlling on the part of marketing professionals. Regarding the parameters estimation, reaching a high level of fitting accuracy is not a trivial task. At this point, mathematical modeling reveals a fruitful tool to maximize the diffusion of marketing contents and minimize the costs of running a campaign.

To sum up, optimal control theory plays a key role on the effective diffusion of viral marketing campaigns, by providing not only higher levels of infection than the obtained without using it, but also by speeding up the transmission process within the target audience.

Acknowledgements The authors would like to acknowledge the comments and suggestions from the reviewers, which improved the quality of the paper. This work was supported in part by the Portuguese Foundation for Science and Technology (FCT - Fundação para a Ciência e a Tecnologia), through CIDMA - Center for Research and Development in Mathematics and Applications, within project UID/MAT/04106/2013; and through Algoritmi R\&D Center, under COMPETE: POCI-010145-FEDER-007043 within the Project Scope: UID/CEC/00319/2013.

\section{References}

1. W.H. Fleming, R.W. Rishel, Deterministic and Stochastic Optimal Control, vol. 1 (Springer Science \& Business Media, New York, 2012)

2. E. Gray, Dove's 'real beauty sketches' ad campaign tells women 'you're more beautiful than you think'. The Huffington Post (2013)

3. H.W. Hethcote, The mathematics of infectious diseases. SIAM Rev. 42(4), 599-653 (2000)

4. K. Kandhway, J. Kuri, How to run a campaign: optimal control of SIS and SIR information epidemics. Appl. Math. Comput. 231, 79-92 (2014)

5. A. Karnik, P. Dayama, Optimal control of information epidemics, in 2012 Fourth International Conference on Communication Systems and Networks (COMSNETS) (IEEE, 2012), pp. 1-7

6. R. Kheirandish, A.S. Krishen, P. Kachroo, Application of optimal control theory in marketing: What is the optimal number of choices on a shopping website? Int. J. Comput. Appl. Technol. 34(3), 207-215 (2009)

7. J.C. Lagarias, J.A. Reeds, M.H. Wright, P.E. Wright, Convergence properties of the neldermead simplex method in low dimensions. SIAM J. Optim. 9(1), 112-147 (1998)

8. S. Lenhart, J.T. Workman, Optimal Control Applied to Biological Models (CRC Press, Boca Raton, 2007)

9. J. Leskovec, L.A. Adamic, B.A. Huberman, The dynamics of viral marketing. ACM Trans. Web (TWEB) 1(1), 1-39 (2007)

10. M. Martcheva, Introduction to Mathematical Epidemiology, vol. 61 (Springer, Berlin, 2015)

11. A. Mochalova, A. Nanopoulos, A targeted approach to viral marketing. Electron. Commer. Res. Appl. 13(4), 283-294 (2014)

12. L.S. Pontryagin, Mathematical Theory of Optimal Processes (CRC Press, Boca Raton, 1987)

13. L. Porter, G.J. Golan, From subservient chickens to Brawny men: a comparison of viral advertising to television advertising. J. Interact. Advert. 6(2), 4-33 (2006) 
14. H.S. Rodrigues, M.J. Fonseca, Can information be spread as a virus? viral marketing as epidemiological model. Math. Methods Appl. Sci. 39(16), 4780-4786 (2016)

15. R.T. Rust, T. Ambler, G.S. Carpenter, V. Kumar, R.K. Srivastava, Measuring marketing productivity: current knowledge and future directions. J. Market. 68(4), 76-89 (2004)

16. S. Schroeder, Youtube now has one billion monthly users (2016)

17. K. Sohn, J.T. Gardner, J.L. Weaver, Viral marketing-more than a buzzword. J. Appl. Bus. Econ. 14(1), 21-42 (2013)

18. L. Stampler, How dove's' real beauty sketches' became the most viral video ad of all time. Business Insider (2013)

19. M. Woerndl, S. Papagiannidis, M. Bourlakis, F. Li, Internet-induced marketing techniques: critical factors in viral marketing campaigns. Int. J. Bus. Sci. Appl. Manag. 3(1), 33-45 (2008) 